\title{
CINQ LEVURES DU GENRE DEBARYOMYCES CONSIDÉRÉES COMME PATHOGÈNES
}

\author{
Par Masao OTA \\ Ancien professeur de dermatologie à la Faculté de médecine de Moukden, \\ Chargé de mission en Europe.
}

Pendant mon voyage, j'ai recueilli, en Amérique, à Paris et en Allemagne, un certain nombre de levures considérées comme pathogènes. Ce sont :

1. - Levure de méningite (à Philadelphie, laboratoire du $\mathrm{D}^{\mathrm{r}}$ Weidman).

2. - Atelosaccharomyces Hudeloi (à Paris, laboratoire de Parasitologie de la Faculté de médecine).

3. - Saccharomyces lithogenes, Sanfelice (cette levure et les suivantes (1) ont été données par le ṕrof. Buschke, de Berlin).

4. - Saccharomyces canis, Sanfelice.

5. - Levure de Kartrick.

6. - Levure de Lavena.

7. - Levure de Læderich.

8. - Levure de Poulman.

9. - Levure de Skutetzky.

10. - Levure de Copelli.

11. - Levure de Lundsgaard.

12. - Levure de Lædsgaard.

13. - Levure de Hildegaard.

14. - Levure de Léopold.

15. - Levure de Bräunli.

16. - Levure de Winternitz.

17. - Levure de Foulerton.

18. - Levure de Busse.

19. - Levure de Curtis.

20. - Levure de Kislovic.

(1) La plupart de ces levures proviennent vraisemblablement de la collection mićrobiologique de Kral (dirigée actuellement par le prof. Pribram). Je les ai obtenues au laboratoire de dermatologie du Virchow-Krankenhaus à Berlin, grâce à l'obligeance du prof. Buschke et du Dr Harry, ce dont je les remercie vivement.

Annales de Parasitologie, T. I, No 2. - Juin 1923. 
M. da Fonseca avait admis autrefois, au laboratoire du professeur Guilliermond, à Lyon, que le soi-disant Atelosaccharomyces Hudeloi de Beurmann et Gougerot, appartenait au genre Debaryomyces. J'ai trouvé, en outre, quatre espèces du genre Debaryomyces parmi les levures de ma collection.

Toutes les autres espèces, à part le Saccharomyces canis qui est du genre Saccharomyces, ne produisent pas de spores. Donc, elles doivent être classées dans le genre Cryptococcus.

J'ai étudié cinq espèces de levures considérées comme pathogènes, appartenant au genre Debaryomyces au laboratoire de Botanique de la Faculté de Lyon, sous la direction du prof. Guilliermond. Ce sont :

1. - Levure d'Hudelo, Duval et Loederich (Atelosaccharomyces Hudeloi).

2. - Levure de Léopold.

3. - Levure de Lundsgaard.

4. - Levure de Lædegaard.

5. - Levure de Hildegaard.

\section{I. - LEVURE DE HUDELO}

Debaryomyces Hudeloi (de Beurmann et Gougerot, 1909) da Fonseca emend., 1922.

Syn.: Atelosaccharomyces Hudeli de Beurmann et Gougerot, 1909.

1. Origine. - Cette levure a été isolée, en 1906, par Hudelo, Rubens-Duval et Lœderich, à Paris, d'un cas de blastomycose à foyers multiples, du type Busse-Buschke. La souche qui a servi de point de départ au travail de da Fonseca et au mien est conservée dans la mycothèque du laboratoire de Parasitologie de la Faculté de médecine de Paris.

2. Morphologie. - Sur moût gélosé à $25^{\circ}$, au bout de 24 heures, les cellules sont généralement ovoïdes ou rondes, mais parfois oblongues. Elles peuvent rester isolées, mais, le plus souvent, elles sont réunies deux à deux, ou plus rarement en chaînettes. Le nombre des cellules de ces chaincttes est ordinairement de quatre ou cinq. Le diamètre des cellules varie de 2 à $4 \mu$. Si les cellules sont oblongues, leur longueur peut atteindre $8 \mu$. On observe rarement des éléments géants, de forme irrégulière : un d'eux mesurait $16 \mu$ de longueur et $6 \mu$ de largeur à son plus grand diamètre. Une quantité de cellules contiennent déjà un petit globule de graisse (1, fig. 1). 
Sur le même milieu à $35^{\circ}$, au bout de deux jours, les cellules sont généralement plus grandes qu'à $25^{\circ}$. Elles ont une tendance à se grouper. Les chaînettes sont pourvues quelquefois de ramifications latérales. La dimension des cellules d'une chaînette est souvent inégale. Parfois on trouve des cellules géantes de forme irré-

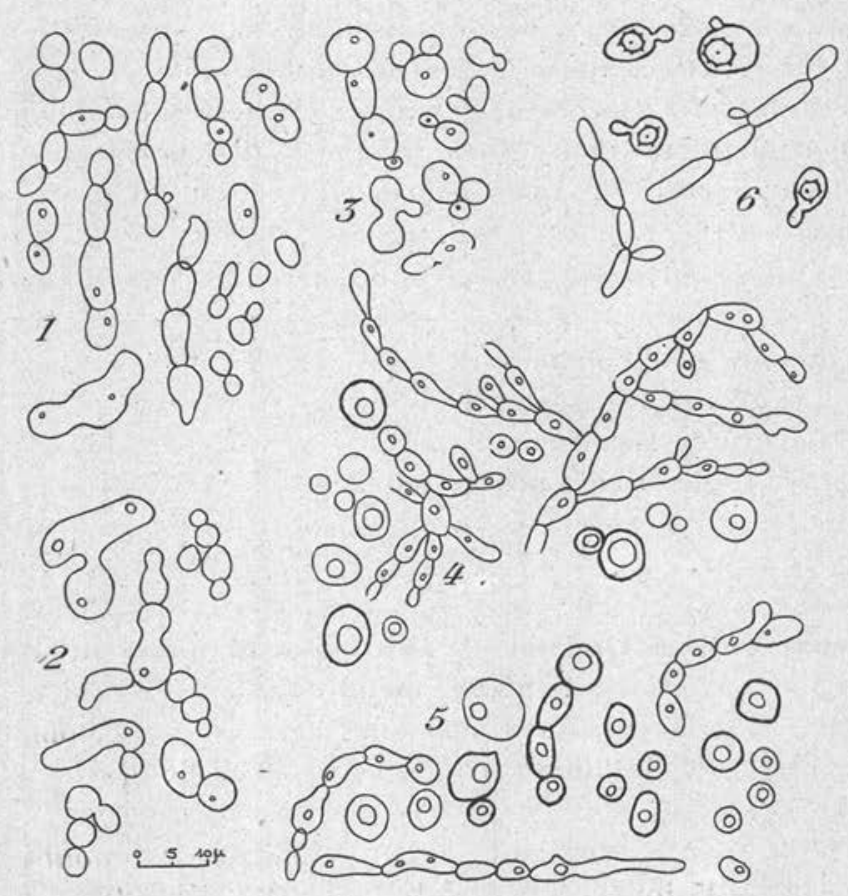

Fig. 1. - Lerure de Hudelo (Atelosaccharomyces Hudeloi). - 1, cellules d'une culture sur moût gélosé à $25^{\circ}$, au bout d'un jour; 2 , cellules d'une culture sur moût gélosé à $35^{\circ}$, au bout de 2 jours; 3 , cellules d'une culture sur carotte à $25^{\circ}$, au bout d'un jour ; 4 , cellules de la même culture à $25^{\circ}$, au bout d'un mois ; 5 , cellules d'une culture sur moût gélosé à $25^{\circ}$, au bout de 40 jours ; 6 , cellules d'une culture sur gélose de Gorodkowa à $25^{\circ}$, au bout de 7 jours.

gulière. Dans la plupart des éléments, on remarque un petit globule graisseux (2, fig. 1).

Quand la culture vieillit, les cellules unies en chainettes augmentent de nombre et deviennent généralement plus volumineuses : elles sont souvent pourvues d'une membrane épaisse. Le diamètre de cellules âgées de 25 jours arrive jusqu'à 7 ou $8 \mu$ ( 5 , fig. 1 ).

Sur tranches de carotte à $25^{\circ}$, au bout de 24 heures, l'aspect microscopique des cellules est à peu près le même que sur moût 
gélosé au même âge (3, fig. 1). Mais dans la culture d'un mois, on trouve les chaînettes de cellules beaucoup plus développées : elles se composent d'éléments allongés et ovoïdes, et possèdent des ramifications latérales formées de plusieurs cellules. La plupart des cellules, cependant, sont isolées ou souvent groupées deux à deux. Dans ce cas, elles sont généralement de grande dimension. En général, quand la culture vieillit, les cellules contiennent un ou plusieurs globules graisseux de grandeur remarquable (4, fig. 1).

3. Caractères de végétation sur moût de bière liquide. - La levure forme, à $25^{\circ}$, un dépôt d'un blanc brunâtre. Elle ne produit ni voile, ni anneau au bout de 30 jours.

4. Température maxima pour le bourgeonnement. - La levure produit un faible développement à $37^{\circ}$, elle ne pousse déjà plus à $39^{\circ}$. La température maxima est située entre 37 et $39^{\circ}$. La température optima semble être au voisinage de $30^{\circ}$. Elle pousse au voisinage de $5^{\circ}$. A $12^{\circ}$, le développement est abondant.

5. Sexualité et sporulation. - Dans cette levure, la sporulation est en général très rare. Cultivée sur gélose de Gorodkowa, au bout de 7 jours, il y a si peu d'asques, qu'il faut un examen minutieux pour les trouver.

La copulation s'effectue entre deux cellules de dimensions généralement inégales. Le gamète mâle est ordinairement beaucoup plus petit que le gamète femelle. Les asques ne renferment qu'une seule spore. Les ascospores sont rondes avec un petit globule graisseux au centre et ont une membrane pourvue de petites protubérances. Le diamètre de ces ascospores est $2-3 \mu$ (6, fig. 1).

6. Colonie géante sur moût gélosé et culture sur moût gélatiné. - Au bout de 35 jours, la colonie est d'un blanc grisâtre, brillant ; quelques rayons partent du centre ; les bords sont nets, avec quelques échancrures. Colonie de $3 \mathrm{~cm}$. sur moût gélatiné, on ne constate pas de liquéfaction au bout de 36 jours.

7. Caractères biochimiques. - La levure invertit le saccharose. Par la méthode des petites fermentations de Lindner, elle a donné une fermentation assez forte du saccharose et du glycose. Elle n'a montré aucune action sur le levulose, le maltose, le galactose, le lactose et le mannose.

8. Action pathogène sur le cobaye. - Un cobaye, après inoculation sous-cutanée et intrapéritonéale avec une assez grande quantité d'émulsion de cette levure, a montré un abcès à la région de l'inoculation. Une douzaine de jours après, l'abeès a disparu spontanément et l'animal a guéri. L'autopsie n'a pas été faite. 
9. Affinités. - La levure, par ses asques dérivés de copulations hétérogamiques et ieur spore unique à membrane verruqueuse, présente les caractères du genre Debaryomyces (Klöcker).

\section{II. - LEVURE DE LEOPOLD}

\section{Debaryomyces Leopoldi $\mathrm{n}$. sp.}

1. Origine. - Cette levure a été isolée en 1900 par Léopold, à Dresde, d'un carcinome de l'ovaire (Kœenigl. Frauenklinik).

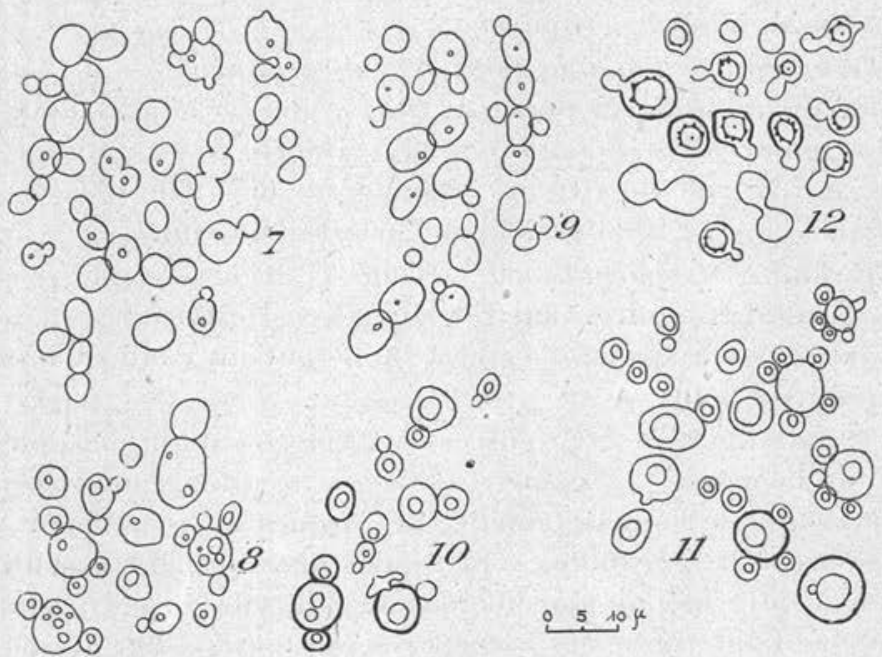

Fig. 2. - Levure de LÉopold. - 7, cellules d'une culture sur moût gélosé à $25^{\circ}$, au bout d'un jour ; 8 , cellules de la même culture à $25^{\circ}$, au bout de 9 jours; 9 , cellules d'une culture sur carotte à $25^{\circ}$, au bout d'un jour; 10 , cellules d'une culture sur moût à $25^{\circ}$, au bout de 40 jours ; 11 , cellules d'une culture sur carotte à $25^{\circ}$, au bout d'un mois ; 12, copulation et formation des asques dans une culture sur gélose de Gorodkowa à $25 \%$, au bout de 9 jours.

2. Morphologie. - Sur moût gélosé à $25^{\circ}$, au bout de 24 heures, les cellules sont généralement rondes ou ovoïdes. Leur petit diamètre varie de 3 à $5 \mu$. On trouve parfois des grands éléments qui mesurent par exemple $6 \times 7 \mu$. Les cellules sont solitaires ou groupées par deux, mais elles montrent aussi une tendance à se réunir en amas plus nombreux. Une partie d'entre elles contiennent déjà un petit globule graisseux ( 7 , fig. 2). La formation de chaînettes composées de nombreuses cellules, comme dans l'espèce précédente, n’a jamais été observée.

Dans la même culture, les cellules deviennent généralement plus 
grandes au bout de 9 jours ; en même temps, on 'y constate la présence d'un ou plusieurs globules qui s'agrandissent. Une de ces grosses cellules mesure $6 \times 8 \mu$ ( 8 , fig. 2$)$. Au bout de 40 jours, la membrane d'un certain nombre d'entre elles s'épaissit et les globules graisseux deviennent encore plus volumineux. La forme des cellules cependant ne montre pas de changement remarquable (10, fig. 2).

Sur tranches de carotte, l'aspect est à peu près identique (9, fig. 2$)$. Au bout d'un jour, on voit des cellules ovoïdes ou rondes, avec ou sans globule graisseux, solitaires ou groupées par deux ou parfois en plus grand nombre. Un certain nombre de grosses cellules portent autour d'elles quelques cellules beaucoup plus petites. Au bout d'un mois, une quantité de grandes cellules se montrent dans la culture. La grandeur des globules graisseux est remarquable (11, fig. 2).

Sur aucun milieu, on n'observe de formation mycélienne.

3. Caractères de végétation sur moùt de bière liquide. - La levure n'a donné, au bout de 30 jours, qu'un dépôt blanc brunâtre.

4. Température maxima pour le bourgeonnement. - La levure ne pousse pas à $40^{\circ}$. A $36^{\circ}$, le développement est très faible. Il est douteux à $39^{\circ}$. A $37^{\circ}$ une partie de culture pousse. La température maxima semble être située entre 37 et $40^{\circ}$. A $5^{\circ}$, la levure donne un développement appréciable. A $12^{\circ}$, il s'effectue bien.

5. Sexualité et sporulation. - La sporulation a été observée dans la culture sur gélose de Gorodkowa, au bout de 9 jours. Les asques ont été trouvés en général en petit nombre, plus nombreux, toutefois, que dans la culture de l'espèce précédente. Les asques renferment une seule spore. La dimension des spores est de 2 à $3 \mu$; leur membrane est généralement pourvue de protubérances, parfois celles-ci manquent. Sur bloc de plâtre, j'ai obtenu des asques au bout de 4 jours.

La copulation est ordinairement hétérogamique. J'ai trouvé, cependant, quelques cas de copulation s'effectuant entre deux cellules de dimensions semblables (12, fig. 2).

6. Culture géante sur moût de bière gélosé et cultuie sur moût gélatiné. - Au bout de 30 jours, colonie très développée, blanche, quelques rayons partant du centre, bord net. Sur moût gélatiné, pas de liquéfaction au bout de 35 jours.

7. Caractères biochimiques. - La levure invertit le saccharose. Par la méthode des petites fermentations de Lindner, elle produit une fermentation très légère du glycose. Des traces de fermentation du saccharose, du levulose, du galactose et du mannose sont cons- 
tatées. La fermentation a été négative avec le maltose, le raffinose et le lactose.

8. Action pathogène sur le cobaye. - Par inoculation sous-cutanée et intrapéritonéale, l'animal n'a montré aucun signe pathologique. Une petite tumeur à la région d'inoculation a disparu en quelques jours. L'autopsie n'a pas été faite.

9. Affinités. - Un de mes compatriotes et collègues, le $\mathrm{D}^{\mathrm{r}} \mathrm{M}$. Sasagawa, a étudié la plupart des levures pathogènes ou parasites que j'ai recueillies en Allemagne. Dans son travail il a classé cette espèce avec toutes les espèces ci-après dans le genre Cryptococcus. D'après lui, ce sont des levures asporogènes présentant des ressemblances de formes avec les Torulaspora et les Torula.

Cette classification doit être modifiée à la suite de mes recherches qui m'ont permis, au contraire, de mettre en évidence dans cette levure la présence d'asques. La eopulation hétérogamique qui précède la formation de l'asque et la présence dans chaque asque d'une seule spore à membrane verruqueuse, me permettent de rapporter cette levure, comme la levure d'Hudelo, au genre Debaryomyces Klöcker.

\section{III. - LEVURE DE LUNDSGAARD}

\section{Debaryomyces Lundsgaardi $\mathrm{n}$. sp.}

1. Origine. - Cette levure a été isolée, en 1900, par Lundsgaard, à Copenhague, d'une hypokératite.

2. Morphologie. - Sur moût gélosé à $25^{\circ}$, au bout d'un jour, les cellules sont ovoïdes ou rondes. Elles sont ordinairement de petites dimensions (2,5-3,5 $\mu)$, solitaires ou groupées par deux. Cependant, on trouve un certain nombre de cellules groupées en petites colonies. Parfois on observe un petit globule de graisse (13, fig. 3 ).

Dans la même culture, on constate, au bout de 3 jours, quelques cellules plus volumineuses, mesurant $6 \times 8 \mu$. Les globules graisseux sont déjà agrandis (14, fig. 3 ).

Au bout de 9 jours, j'ai trouvé quelques formations mycéliennes rudimentaires, partant d'une cellule ronde, avec ou sans cloison transversale. Parfois on observe des chainettes d'un certain nombre de cellules, mais moins développées que dans la levure d'Hudelo (15, fig. 3).

Dans une culture sur le même milieu, au bout de 40 jours, se montrent quelques cellules de grandeur remarquable. L'une d'elles, arrondie, avec une membrane mince, contenait des globules graisseux. Sa dimension était $12 \times 15 \mu$. Les grandes cellules portent 
souvent quelques cellules-filles moins grandes autour d'elles (16, fig. 3).

Sur tranches de carotte, la forme et la grandeur des cellules sont presque les mêmes que sur moût gélosé ; les cellules géantes sont, cependant, beaucoup plus nombreuses. Elles sont pourvues d'une membrane épaisse et offrent dans leur intérieur des globules de graisse très volumineux (18, fig. 3 ).

3. Caractères de végétation sur moût de bière liquide. - La
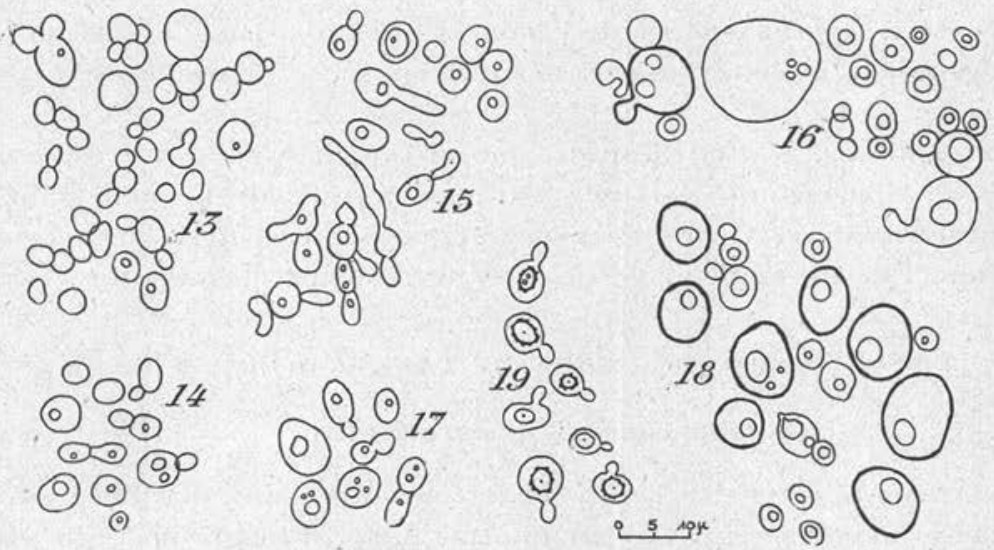

Fig. 3. - Levure de Lundsgannd. - 13, cellules d'une culture sur moût gélosé à $25^{\circ}$, au bout d'un jour; 14 , cellules de la même culture, au bout de 3 jours ; 15 , cellules de la même culture, au bout de 9 jours ; 16 , cellules de la même culture, au bout de 40 jours; 17 , cellules d'une culture sur carotte à $25^{\circ}$, au bout de 5 jours ; 18 , cellules de la même culture, au bout d'un mois ; 19 , formation des asques dans une culture sur gélose de Gorodkowa à $25^{\circ}$, au bout de 12 jours.

levure a donné un dépôt d'un blanc brunâtre. Elle ne forme ni anneau, ni voile, même au bout de 30 jours.

4. Température maxima pour le bourgeonnement. - La levure pousse à $35^{\circ}$, mais pas à $36^{\circ}$. La température maxima est donc située entre $35^{\circ}$ et $36^{\circ}$. A $5^{\circ}$, elle donne un développement appréciable; à $12^{\circ}$ elle végète assez bien.

5. Sexualité et sporulation. - La sporulation, observée sur gélose de Gorodkowa, est rare. Elle est précédée d'une copulation entre deux cellules de dimensions généralement inégales. Le gamète mâle est très petit et souvent allongé. Les asques sont ordinairement petits, ronds ou ovoïdes, mesurant $3-5 \mu$, ils ont une membrane un peu épaisse. Ils renferment une seule spore. Les ascospores ont un petit globule de graisse au centre et une membrane pourvue de protubérances. Leur diamètre était de $2-3 \mu$ (19, fig. 3 ). 
6. Colonie géante sur moût gélosé, culture sur moût gélatiné. Au bout de 35 jours, la colonie est assez grande (diam. 5 cent.), d'aspect brillant, de couleur blanche, avec de fins rayons partant du centre ; le bord est net, avec de rares échancrures. Sur moût gélatiné, pas de liquéfaction au bout de 37 jours.

7. Caractères biochimiques. - La levure invertit le saccharose. Par la méthode des petites fermentations de Lindner, elle ne provoque pas la fermentation du saccharose, du glycose, du levulose, du maltose, du galactose, du raffinose, du lactose et du mannose.

8. Action pathogène sur le cobaye. - Il n'y a pas eu d'action pathogène sur le cobaye après inoculation sous-cutanée et intrapéritonéale.

9. Affinités. - Cette espèce, qui se rapporte au genre Debaryomyces, a beaucoup de ressemblance avec celle de Léopold, elle se différencie de cette dernière surtout par ses caractères de fermentation. Elle est également très voisine des levures suivantes.

\section{IV. - LEVURE DE LAEDEGAARD}

\section{Debaryomyces Laedegaardi $\mathrm{n}$. sp.}

1. Origine. - Cette levure était conservée dans la collection de Kràl, et avait été envoyée par le prof. Bayer d'Innsbrück. Elle était connue comme un agent de maladie cutanée. Je n'ai pas pu avoir connaissance de la description originale.

2. Morphologie. - Sur moût gélosé à $25^{\circ}$, au bout d'un jour, les cellules sont ovoïdes ou rondes, parfois cylindriques. Elles sont solitaires ou groupées par deux ou plus. Le diamètre des éléments arrondis varie de 2,5 à $5 \mu(20$, fig. 4$)$.

Dans la même culture, j'ai trouvé, au bout de 3 jours, que les cellules étaient généralement peu volumineuses et contenaient d'assez gros globules graisseux (21, fig. 4).

Au bout de 9 jours, j'ai observé quelques chaînettes formées de cellules de faible diamètre et généralement allongées. Des asques ont été observés, mais ils étaient très rares (22, fig. 4).

$\mathrm{Au}$ bout de 40 jours, dans les mêmes conditions, les cellules étaient devenues beaucoup plus grosses. On voyait de gros éléments entourés de quelques cellules plus petites (23, fig. 4 ).

Sur tranches de carotte, l'aspect microscopique est le même que sur moût gélosé. La figure 24 (fig. 4) montre des cellules d'une culture de 5 jours. Quand la culture sur carotte vieillit, on constate souvent des cellules de grandes dimensions : une d'elles mesurait $10 \mu$ (25, fig. 4). 
3. Caractères de végétation sur moût de bière liquide. - La levure a donné un dépôt d'un blanc brunâtre. Au bout de 35 jours, elle n'a formé ni voile, ni anneau.

4. Température maxima pour la croissance. - La levure pousse à $35^{\circ}$, mais pas à $37^{\circ}$. Sa température maxima pour la croissance est donc située entre 35 et $37^{\circ}$. A $5^{\circ}$, la levure ne se développe pas de manière appréciable ; elle végète faiblement à $12^{\circ}$.

5. Sexualité et sporulation. - La formation des asques a été observée dans les cultures sur gélose de Gorodkowa, au bout de
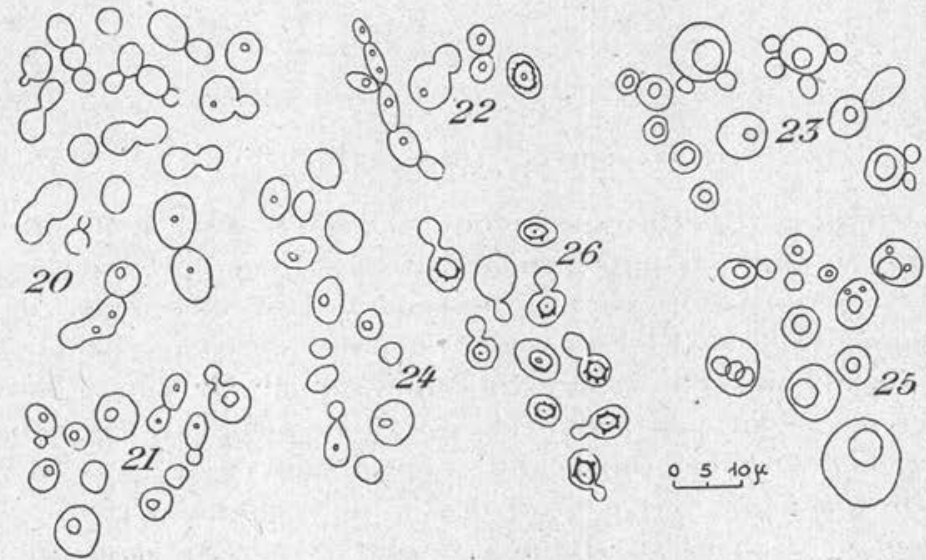

Fig. 4. - Levure de LakdegaArd. - 20, cellules d'une culture sur moût géłosé à $25^{\circ}$, au bout d'un jour: 21 , cellules de la même culture, au bout de 3 jours ; 22 , cellules de la même culture, au bout de 9 jours; 23 , cellules de la même culture, au bout de 40 jours ; 24 , cellules d'une culture sur carotte à $25^{\circ}$, au bout de 5 jours ; 25 , cellules de la même culture, au bout de 30 jours; 26 , formation des asques sur gélose de Gorodkowa au bout de 12 jours.

12 jours. Elle était d'ailleurs très peu active. Les asques étaient ovoïdes ou parfois polygonaux. Ils étaient pourvus ordinairement d'une membrane épaisse. Leur diamètre était de $3,5-4 \mu$; si les asques étaient oblongs, la largeur atteignait 5-6 $\mu$. Le diamètre des ascospores est de $2-2,5 \mu$.

Les asques dérivent de la copulation de deux cellules généralement inégales (26, fig. 4). Ils renferment une seule ascospore à membrane verruqueuse et contenant au centre un petit globule graisseux.

6. Colonie géante sur moût gélosé et culture sur moût gélatiné. Au bout de 25 jours, colonie de $3 \mathrm{~cm}$., d'un blanc jaunâtre, brillant, avec de fines radiations allant du centre à la périphérie. Le bord est net. 
Sur moût gélatiné, aucune liquéfaction au bout d'un mois.

7. Caractères biochimiques. - La levure invertit le saccharose. Par la méthode des petites fermentations, elle donne une légère fermentation du glycose et fait fermenter très faiblement le saccharose. Pas de fermentation avec le levulose, le maltose, le galactose, le lactose et le mannose.

8. Action pathogène sur le cobaye. - Elle n'a pas été constatée pour le cobaye, après inoculation sous-cutanée et intrapéritonéale.

9. Affinités. - Cette levure, par sa copulation et les caractères de ses spores, se rapporte comme les autres au genre Debaryomyces.

\section{V. - LEVURE DE HILDEGAARD}

\section{Debaryomyces Hildegaardi n. sp.}

1. Origine. - Cette espèce était conservée aussi à la collection de Kràl. Je n'ai pas pu cependant arriver à connaître son origine. Elle était considérée comme provenant d'un laboratoire de Copenhague. Le prof. Klöcker a eu l'amabilité de rechercher des renseignements sur cette espèce ainsi que sur la précédente, mais ses recherches n'ont pas abouti. Les levures n'étaient pas connues, même dans le monde médical de Copenhague.

2. Morphologie. - La forme des cellules sur moût gélosé à $25^{\circ}$, au bout de 24 heures, est ronde, ovoïde et parfois assez allongée. Les cellules se trouvent solitaires ou groupées généralement deux à deux. On trouve aussi des chaînettes composées d'un petit nombre de cellules $(27$, fig. 5$)$.

Au bout de 8 jours, beaucoup de cellules s'agrandissent considérablement. Les cellules les plus grandes ont la dímension de $7 \mu$ (28, fig. 5).

Au bout de 40 jours, les cellules ont généralement une membrane épaisse et des gros globules graisseux (29, fig. 5).

Les grands éléments sont beaucoup plus nombreux sur tranches de carotte, surtout dans les vieilles cultures. Leur dimension atteint souvent $10 \mu$. Elles contiennent des globules graisseux très volumineux (32, fig. 5$)$.

3. Caractères de végétation sur moût de bière liquide et sur moût gélatiné. - La levure a donné un dépôt blanc brunâtre. Il ne se forme ni voile, ni anneau, au bout d'un mois. Sur moût gélatiné, il n'y a pas de liquéfaction au bout de 35 jours.

4. Température maxima pour le bourgeonnement. - A $36^{\circ}$ la levure pousse, mais non à $37^{\circ}$. La température maxima est donc 
située entre $36^{\circ}$ et $37^{\circ}$. A $5^{\circ}$, la levure végète à peine ; à $12^{\circ}$ elle donne un développement très net.

5. Sexualité et sporulation. - Le mode de copulation est généralement hétérogamique. J'ai trouvé cependant quelques cas d'isogamie (33, fig. 5). J'ai observé la formation des asques sur gélose de Gorodkowa : au bout de 9 jours, elle était peu active. Les asques ne renferment qu'une seule ascospore. Les ascospores sont entourées d'une membrane pourvue de protubérances. Elles mesurent

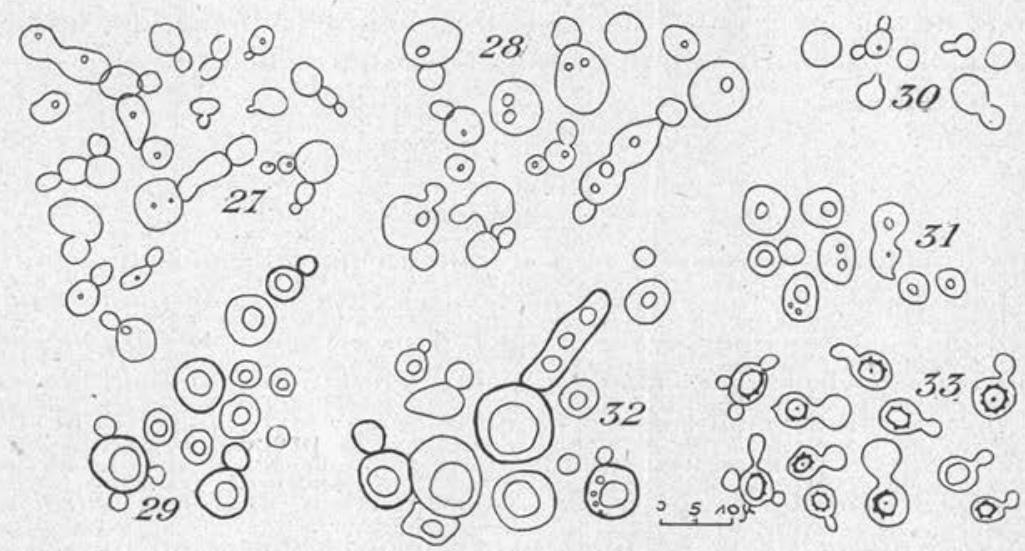

Fig. 5. - Levure de HildegaARd. - 27, cellules d'une culture sur moût gélosé à 25, au bout de 24 heures ; 28 , cellules de la même culture, au bout de 8 jours ; 29 , cellules de la même culture, au bout de 40 jours ; 30 , cellules d'une culture sur carotte à $25^{\circ}$, au bout de 24 heures; 31 , cellules de la même culture, au bout de 5 jours ; 32 , cellules de la même culture, au bout d'un mois ; 33 , sporulation et formation des asques sur moût de Gorodkowa, au bout de 9 jours.

2,5-3,5 $\mu$ de diamètre, et renferment au centre un petit globule graisseux.

6. Caractères de végétation sur moùt gélosé. - La levure végète très bien sur moût gélosé à $25^{\circ}$. Au bout d'un jour, on voit apparaître des colonies d'un blanc grisâtre, brillantes.

7. Caractères biochimiques. - La levure invertit le saccharose. Elle donne une légère fermentation avec le saccharose, le glycose, le levulose et le maltose ; elle n'agit pas sur le galactose, le raffinose, le lactose et le mannose.

8. Action pathogène sur le cobaye. - La levure ne s'est pas montrée pathogène pour le cobaye.

9. Affinités. - Cette levure appartient aussi au genre Debaryomyces. 


\section{$* *$}

Il est intéressant de constater que toutes ces levures appartiennent au genre Debaryomyces, créé en 1908 par Klöcker pour D. globosus. A la suite du travail de Klöcker, un grand nombre d'espèces appartenant à ce genre ont été trouvées dans l'air, sur le fromage, sur les saucissons en voie de maturation et dans des exsudats pathologiques. Le genre Debaryomyces est donc très répandu.

Je tiens, en terminant ce travail, à remercier M. le prof. Guilliermond de son obligeante direction et M. le prof. Arloing, qui a bien voulu me permettre d'utiliser son laboratoire pour expérimenter sur les animaux.

\section{RÉSUMÉ}

Ce travail est consacré à la description complète, morphologique et biologique de cinq espèces de levures. Les caractères de leurs ascospores permettent de les ranger dans le genre Debaryomyces Klöcker. Debaryomyces Hudeloi a déjà été étudié par da Fonseca et mes résultats confirment l'opinion de ce mycologue. Pour les quatre autres levures non nommées, je propose les noms de Debaryomyces Leopoldi n. sp., D. Lundsgaardi n. sp., D. Laedegaardi n. sp., D. Hildegaardi n. sp. Bien qu'isolées de lésions humaines, ces cinq espèces se sont montrées très faiblement pathogènes. Ce travail montre en outre que le genre Debaryomyces est bien plus répandu qu'on ne le croyait tout d'abord.

\section{Bibliographie}

Fonseca (da). - Sobre os agentes das blastomycoses europeas. Cyclo sexuado e posiçao systematica do Levedo de Hudelo. Brazil-Medico, XXXVI, II, 1922.

Leopold. - Untersuchungen zur Aetiologie des Carcinoms und ueber die pathogenen Blastomyceten. Arch. $f$. Gynaekologie, LXI, 1900.

Lundsgando. - Klin. Monatshefte für Augenheilkunde, XXXVIII, 1900.

SASAGAwa. - Zur Systematik pathogener und parasitischer Hefen. Morphologischbiochemischen Studie. Centralbl.f. Bakt., I Abt. Originale, LXXXVIII, 1922, p. 269. 